\title{
Expression of human peroxiredoxin isoforms in response to cervical carcinogenesis
}

\author{
KIYOON KIM ${ }^{1}$, MIRAN YU ${ }^{1}$, SEULHEE HAN ${ }^{1}$, INKYUNG OH ${ }^{1}$, YOUNG-JUN CHOI ${ }^{2}$, \\ SUNGSOO KIM $^{1}$, KYUNGSIK YOON ${ }^{1}$, MINHYUNG JUNG ${ }^{2}$ and WONCHAE CHOE ${ }^{1}$

\begin{abstract}
${ }^{1}$ Department of Biochemistry and Molecular biology, Medical Science and Engineering Research Center for Bioreaction to Reactive Oxygen Species, Biomedical Science Institute, Kyunghee University School of Medicine, Seoul 130-701;

${ }^{2}$ Department of Obstetrics and Gynecology, School of Medicine, Kyung Hee University, Seoul 130-701, Korea
\end{abstract}

Received December 19, 2008; Accepted February 6, 2009

DOI: $10.3892 /$ or_00000366

\begin{abstract}
Despite considerable progress in understanding the function of peroxiredoxin (Prx) in cancer, its expression patterns have not been extensively studied in response to cervical carcinogenesis. We evaluated the expression of Prx isoforms in normal tissue, cervical intraepithelial neoplasia (CIN1, CIN2, and CIN3), and cervical cancer. We found strong pattern of increased Prx II and III immunostaining with increasing severity of the lesion. No difference in staining intensity by grade of lesion was observed for Prx I, and IV. Therefore, we conclude that Prx II and III are upregulated in response to the development of cervical cancer.
\end{abstract}

\section{Introduction}

Cervical cancer ( $\mathrm{Cx} \mathrm{ca}$ ) is the second most common cancer among women worldwide, with a half-million new cases and over 270,000 deaths annually. Cx ca is mainly said to be mediated by Human Papilloma Virus (HPV), and recently published data also revealed a role of oxidative stress in $\mathrm{Cx}$ ca (1). Since the invasive disease is preceded by pre-malignant cervical intraepithelial neoplasia (CIN), it will be important to detect the pre-malignnant stage and treat it suitably to prevent $\mathrm{Cx}$ ca. Oxidative stress affects cells as a result of one of three factors: an increase in oxidant generation, a decrease in antioxidant protection, or a failure to correct oxidative damage. Oxidative stress causes cellular damage and many diseases including aging by impairing cellular functions. It is well-known that anti-oxidative enzymes, such as superoxide dismutase (SOD), catalase, and glutathione peroxidase (GPX), scavenge reactive oxygen species (ROS) and, consequently, protect cells against oxidative stress.

Correspondence to: Dr Wonchae Choe, Department of Biochemistry and Molecular Biology, School of Medicine, Kyung Hee University, \#1, Hoegi-dong, Dongdaemoon-gu, Seoul 130-701, Korea

E-mail:wchoe@khu.ac.kr

Key words: peroxireoxin, reactive oxidative species, cervical cancer
One of the family proteins with an antioxidative function, peroxiredoxin (Prx), so far has revealed six members in mammals (2-4). These enzymes share a common reactive Cys residue in the $\mathrm{N}$-terminal region, and are capable of breaking down $\mathrm{H}_{2} \mathrm{O}_{2}$ as a peroxidase and involve thioredoxin and/or glutathione as the electron donor. Since the sulfhydryl group is the most sensitive to the oxidizing effects of ROS among the amino acid side chains in protein, it is often involved in the intracellular transduction machinery of redox signals in response to physiological and oxidative stimuli $(5,6)$.

Prx isoforms I-IV have an additional Cys residue in the conserved C-terminal region, and are highly homologous (60-80\%) as judged by the amino acid sequence similarity. Prx V also contains an additional Cys in its C-terminal region, which is less conserved (less than 20\%). Prx VI has only one unique Cys. Prx isoforms I-IV belong to the thioredoxin peroxidase subfamily, since they require the small redox protein thioredoxin (Trx). Prx V or Prx VI use other cellular reductants, such as glutathione, for its peroxidase activity. These Prx family members are distributed in the cytosol, mitochondria, peroxisome and plasma, all of which are potential sites of ROS production. However, a lot of biological functions, not directly related to peroxidase activity, have also been reported for the Prx family. It would be expected that these functions might not necessarily depend on peroxidase activity and, therefore, it seems likely that the divergence is due to the unique molecular characteristics intrinsic to each member. There are a few reports that even in cancer cells, Prx also would perform function to protect from oxidative stress, and therefore cancer therapy might be enhanced by inhibiting Prx in cancer cells. Many researchers have reported Prx over-expression in various types of malignant cancer cells $(7,8)$. So far, the pathophysiological significance of Prx proteins, especially in cervical neoplasia, has not been defined. Therefore, we investigated expression of the Prx isoforms (Prx I-IV) to determine whether it is associated with cancer progression in cervical neoplasia.

\section{Materials and methods}

Patient follow-up, tissue array material. Patient materials for tissue array blocks were retrieved from the files of the 
Table I. Information on cases analyzed by immunohistochemistry.

Histopathological diagnosis

\begin{tabular}{cc}
\hline Normal & 42 \\
CIN $^{\mathrm{a}}$ & 92 \\
I & 31 \\
II & 18 \\
III & 29 \\
CIS $^{\mathrm{b}}$ & 14 \\
Cx ca $^{\mathrm{c}}$ & 68 \\
Total $^{2}$ & 202 \\
\hline
\end{tabular}

${ }^{\mathrm{a} C e r v i c a l}$ intraepithelial neoplasia; ${ }^{\mathrm{b}}$ cervical carcinoma in situ; cervical cancer.
Department of Gynecologic Oncology at Kyung-Hee Medical Center. Two hundred and two patients diagnosed by immunohistochemistry and 9 patients by immunoblotting, who had cervical cancer and underwent surgery at KyungHee Medical Center for preinvasive and invasive cervical cancer, were enrolled in this study. Each sample was classified into 3 groups: normal, cervical intraepithelial neoplasia (CIN) and Cx ca.

CIN is the abnormal growth of potentially precancerous cells in the cervix. To tell the difference of developing cancer stage, it was categorized histologically as CIN 1, CIN2, and CIN3, depending on the severity of the lesions (1). CIN1 (grade I), the least risky type, represents only mild dysplasia $(9,10)$. It is confined to the basal $1 / 3$ of the epithelium. CIN2 (grade II) is moderate dysplasia confined to the basal $2 / 3$ of the epithelium, and CIN3 (grade III) is severe dysplasia that spans $>2 / 3$ of the epithelium, and may involve the full thickness. Carcinoma in situ (CIS) is non-invasive cancer which has not grown into deeper tissues of the cervix.
A)

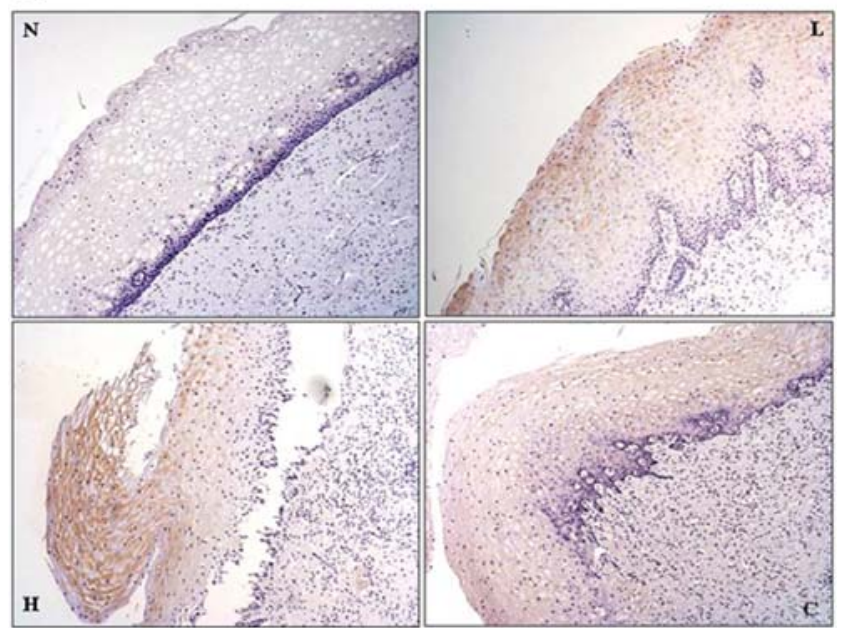

C)

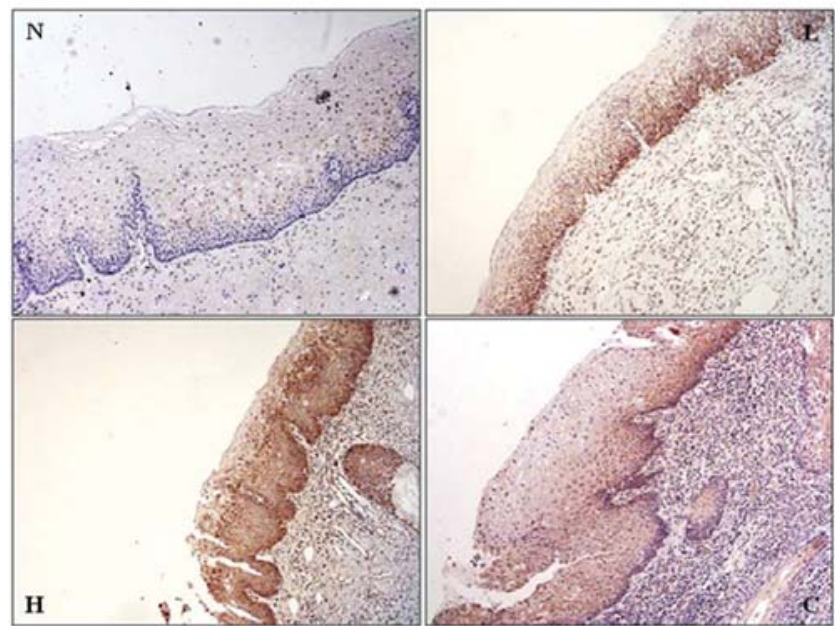

B)

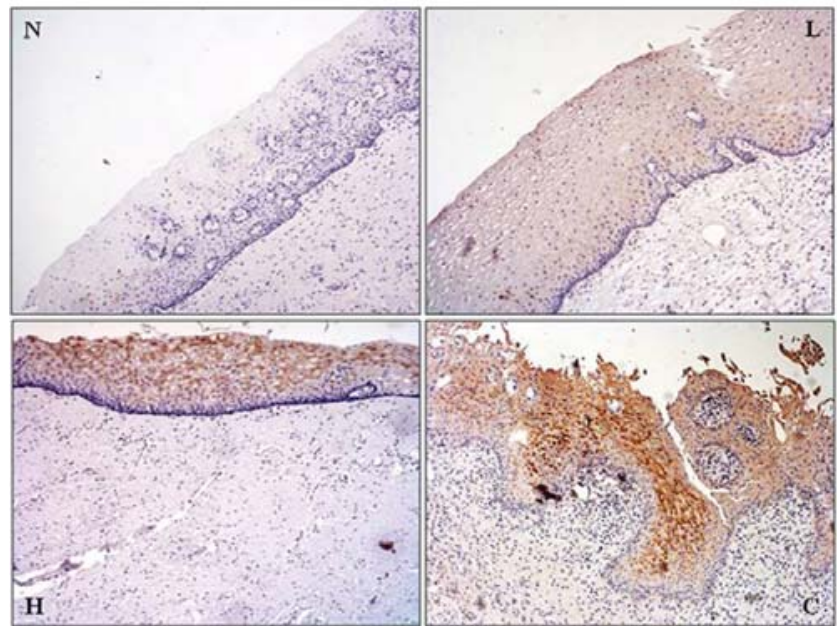

D)

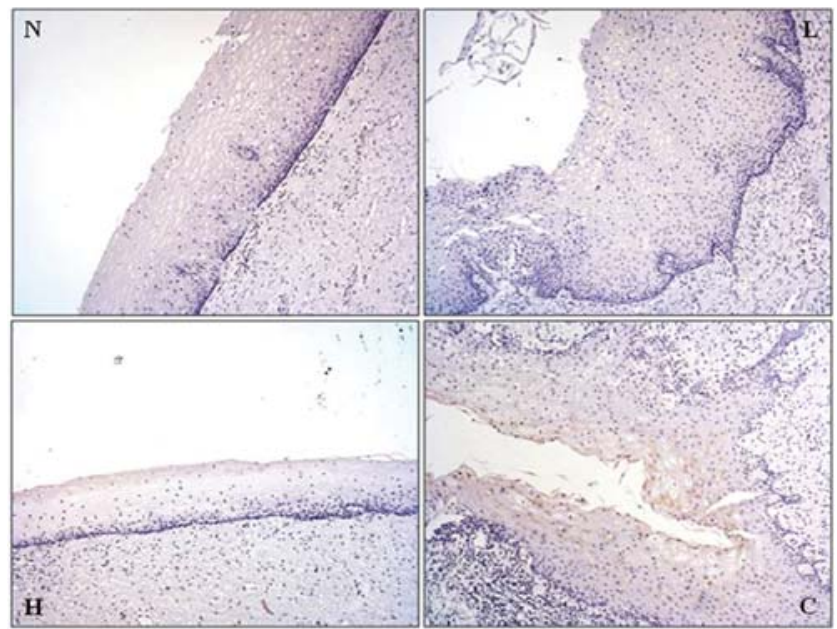

Figure 1. Immunohistochemical staining of Prx isoforms in human cervical tissues. (A) Prx I, (B) Prx II, (C) Prx III, (D) Prx IV. Each Prx families (A, B, C and D) were immunostained in normal (N), low-grade CIN (L), high-grade CIN (H), Cx ca (C). Low-grade CIN means CIN I and II stage, high-CIN means CIN III and CIS stage. 
Table II. Intensity of Prx immunostaining in normal, CINs and cervical cancers.

\begin{tabular}{|c|c|c|c|c|c|}
\hline Prx isoform & Cervical specimen & - & + & ++ & +++ \\
\hline \multirow[t]{4}{*}{ I } & Normal & $6 / 12(50.0)$ & $0 / 12(0.0)$ & $6 / 12(50.0)$ & $0 / 12(0.0)$ \\
\hline & Low-grade CINs ${ }^{a}$ & 1/9 (11.1) & $6 / 9(66.7)$ & 1/9 (11.1) & $1 / 9(11.1)$ \\
\hline & High-grade CINs ${ }^{b}$ & $5 / 8(62.5)$ & $3 / 8(37.5)$ & $0 / 8(0.0)$ & $0 / 8(0.0)$ \\
\hline & $\mathrm{Cx} \mathrm{ca}^{\mathrm{c}}$ & $6 / 20(33.3)$ & $12 / 20(60.0)$ & $2 / 20(16.6)$ & $0 / 20(0.0)$ \\
\hline \multirow[t]{4}{*}{ II } & Normal & 8/10 (80.0) & $1 / 10(10.0)$ & $1 / 10(10.0)$ & $0 / 10(0.0)$ \\
\hline & Low-grade CINs & $0 / 10(0.0)$ & $5 / 10(50.0)$ & $3 / 10(30.0)$ & $2 / 10(20.0)$ \\
\hline & High-grade CINs & $2 / 9(22.2)$ & $2 / 9(22.2)$ & 4/9 (44.4) & $1 / 9(11.1)$ \\
\hline & $\mathrm{Cx} \mathrm{ca}$ & $3 / 16(18.8)$ & $3 / 16(18.8)$ & $4 / 16(25.0)$ & $6 / 16(37.5)$ \\
\hline \multirow[t]{4}{*}{ III } & Normal & $5 / 10(50.0)$ & $1 / 10(10.0)$ & 4/10 (40.0) & $0 / 10(0.0)$ \\
\hline & Low-grade CINs & $1 / 15(6.7)$ & 1/15 (6.7) & $10 / 15(66.7)$ & $3 / 15(20.0)$ \\
\hline & High-grade CINs & $0 / 14(0.0)$ & 4/14 (28.6) & 8/14 (57.1) & $2 / 14(14.3)$ \\
\hline & $\mathrm{Cx} \mathrm{ca}$ & $1 / 20(5.0)$ & $4 / 20(20.0)$ & $8 / 20(40.0)$ & $7 / 20(35.0)$ \\
\hline \multirow[t]{4}{*}{ IV } & Normal & $8 / 10(80.0)$ & $2 / 10(20.0)$ & $0 / 10(0.0)$ & $0 / 10(0.0)$ \\
\hline & Low-grade CINs & $7 / 15$ (46.7) & $5 / 15(33.3)$ & $3 / 15(20.0)$ & $0 / 15(0.0)$ \\
\hline & High-grade CINs & 2/12 (16.7) & $6 / 12(50.0)$ & 4/12 (33.3) & $0 / 12(0.0)$ \\
\hline & $\mathrm{Cx} \mathrm{ca}$ & 4/12 (33.3) & $6 / 12(50.0)$ & 2/12 (16.7) & $0 / 12(0.0)$ \\
\hline
\end{tabular}

${ }^{\mathrm{a} C e r v i c a l}$ intraepithelial neoplasia (CIN) grades I and II; ${ }^{\mathrm{b}}$ cervical intraepithelial neoplasia (CIN) grades III and CIS; ${ }^{\mathrm{c}}$ cervical cancer. Bold text, the strong patterns of increased Prx II and Prx III immunostaining correspond with increasing severity of the cervical cancer.

Immunohistochemistry. The expression levels of peroxiredoxin isoforms were determined by immunohistochemistry. Paraffinembedded specimens were fixed in $4 \%$ buffed formalin. Tissues were sectioned, and attached on a glass slide (thickness, $4 \mu \mathrm{m}$ ). Then, they were deparaffinized in xylene and rehy-drated through ethanol and distilled water. Endogenous peroxidase was blocked with $0.3 \%$ hydrogen peroxide for $10 \mathrm{~min}$. Then they were immersed in $10 \mathrm{mM}$ citric acid monohydrate ( $\mathrm{pH}$ 6.0) for $8 \mathrm{~min}$, and boiled in a microwave oven at $850 \mathrm{~W}$. Specimens were chilled in ice for 20 min. Samples were blocked by protein block serum-free media for $20 \mathrm{~min}$. The specimens were incubated overnight at $4{ }^{\circ} \mathrm{C}$ with a monoclonal antibody against Prx I, II, III, or IV (Ab frontier, Korea) in a dilution of 1:500. The immunostained section was visualized by the Dako EnVision Detection Kit (Dako, Denmark).

Immunoblotting. Tissues from 9 patients were homogenized with 300-500 $\mu$ 1 RIPA buffer (1\% NP-40, 1\% sodium deoxycholate, $0.1 \%$ SDS, $0.15 \mathrm{M} \mathrm{NaCl}, 0.01 \mathrm{M}$ sodium phosphate). Whole lysates were electrophoresed for $2 \mathrm{~h}$ in $14 \%$ SDS-gel. After separating, proteins were transfered to a nitrocellulose membrane. Each membrane was overnight incubated with a monoclonal antibody against Prx I, II, III, or IV (dilutions 1:1000). Following 45-min incubation with secondary antibodies, proteins were visualized by enhanced Western Blotting Luminol Reagent (Santa Cruz Biotechnology, Inc., CA, USA). Protein expression levels were quantified by densitometry.

Statistical analysis. Statistical analysis was performed with SPSS for Windows 11.5. Associations were determined using a 2-tailed t-test, Fisher's exact probability test and
Pearson's correlation. Agreement of the double evaluation was calculated by Cohen's k-correlation analysis. $\mathrm{p} \leq 0.05$ was considered statistically significant.

\section{Results}

Prx I. We examined expression of Prx I by immunohistochemistry and immunoblotting. For this assay, we used 49 tissue samples from patients (normal sample, 12; low-grade CIN, 9; high-grade CIN, 8; Cx ca, 20). As shown in Fig. 1 and Table II, the normal tissues were negative or moderately positive for Prx I. In both low-grade CINs and high-grade CINs, Prx I expression levels were negative or weakly positive. In $\mathrm{Cx}$ ca samples, $60 \%$ were only weakly positive. Immunoblot analysis with patient tissue samples did not show any noteable difference between normal and cancer samples (Figs. 2 and 3).

Prx II. We performed immunohistochemisty with Prx II antibody (normal sample, 10; low-grade CIN, 10; high-grade CIN, 9; Cx ca, 16). As shown in Fig. 1 and Table II, in the normal tissues $80.0 \%$ were negative or undetectable for Prx II. In low-grade CINs, $50.0 \%$ were weakly positive $(\mathrm{P}=0.0047)$. In high-grade $\mathrm{CINs}$ and $\mathrm{Cx}$ ca samples, $44.4 \%$ $(\mathrm{P}=0.0047)$ and $37.5 \%(\mathrm{P}=0.0005)$ were moderately and strongly positive for Prx II, respectively. These results suggest the strong pattern of increased Prx II immunostaining with increasing severity of the lesion during cervical cancer development. Moreover, immunoblotting with patient tissue samples with PrxII antibody showed overexpressed Prx II in most cervical cancer tissues compared with normal tissue (Figs. 2 and 3). 


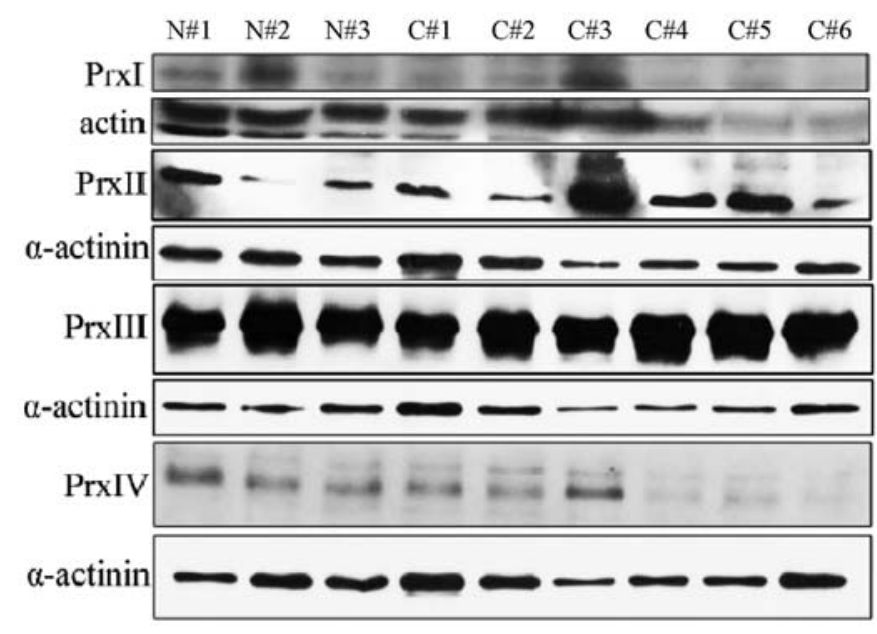

Figure 2. Immunoblot analysis of Prx I, Prx II, Prx III and Prx IV in cervical tissues. A $30 \mathrm{kDa}$ protein band corresponding to the Prx I, Prx II, Prx III or Prx IV was observed using each antibody. 'N' represents normal tissue homogenates from patient and ' $\mathrm{C}$ ' represents the tissue homogenates from cervical carcinoma patients. Actin and $\alpha$-actinin were used as a loading control.
Prx III. To determine whether expression level of Prx III is associated with cervical cancer development or not, we also performed immunohistochemistry in patient tissue samples with Prx III antibody. As shown in Table II and Fig. 1, in $50.0 \%$ normal tissue, Prx III expression was undectable. Most of low-grade CIN group tissues were positive for Prx III $(\mathrm{P}=0.0022)$. In high-grade $\mathrm{CINs}$ and $\mathrm{Cx}$ ca, 57.1\% ( $\mathrm{P}=0.004)$ and $40.0 \%(\mathrm{P}=0.001)$ were positive for Prx III, respectively. Also our immunoblotting results with Prx III antibody showed that, Prx III was about four times more highly expressed in most of patients' cervical cancer tissues compared with normal tissues, although it was easily detected even in normal tissues (Figs. 2 and 3), suggesting that Prx III is upregulated during the cervical cancer development.

Prx IV. We performed immunohistochemisty with Prx IV antibody. In normal group and low-grade CIN group, $80 \%$ and $46.7 \%(\mathrm{P}=0.032)$ were undetected for Prx IV, respectively. In high-grade CINs, 50.0 and $33.3 \%$ were weakly positive and positive, respectively $(\mathrm{P}=0.0006)$. However, in $\mathrm{Cx}$ ca, only $50 \%$ were weakly positive $(\mathrm{P}=0.011)$. Also in immuno-
A)

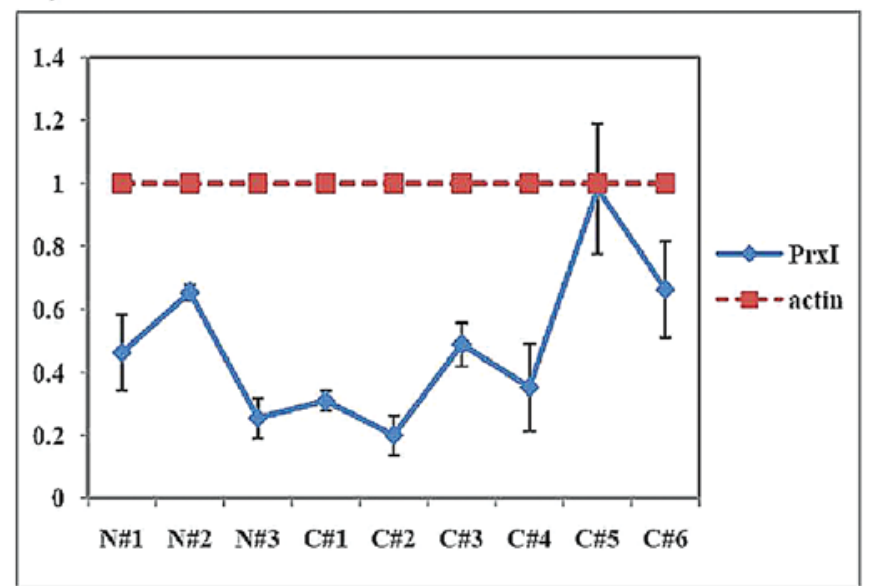

C)

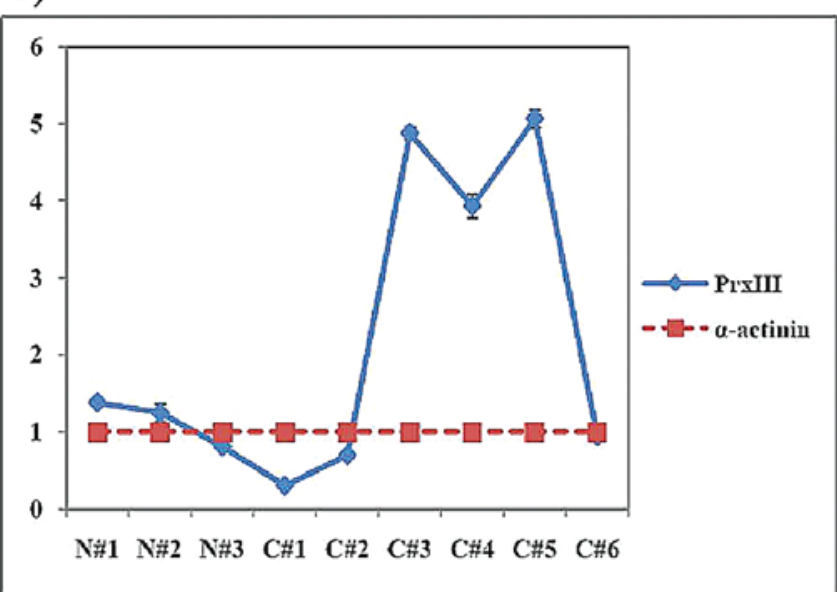

B)

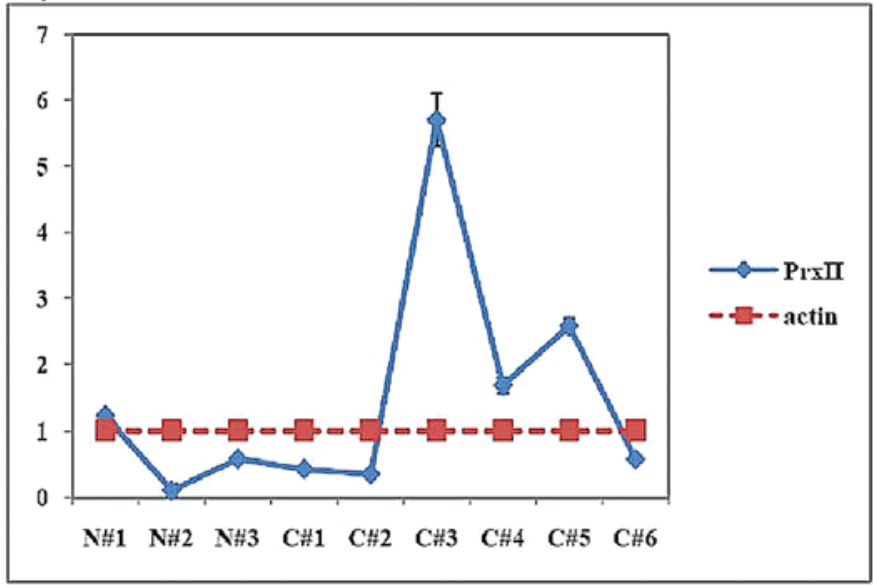

D)

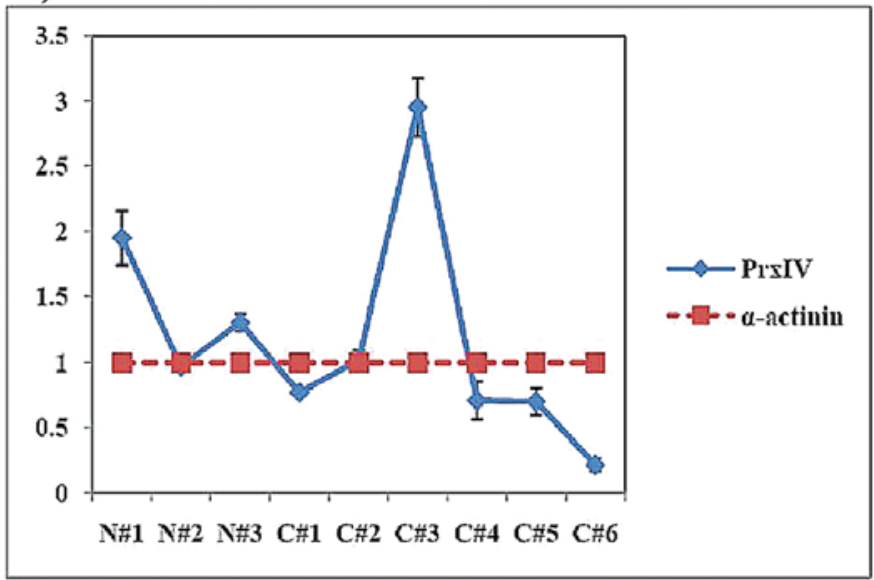

Figure 3. Densitometric analysis of the immunoblotting for expression of Prx isoforms. Intensities of the immunoblotting results were quantitated by densitometry. The expression levels of Prx isoforms were determined relative to that of actin and $\alpha$-actinin. A solid line represents each Prx expression level, and a dotted line represents a standard protein level. Similar data were obtained in several independently performed experiments (mean \pm SD; $\mathrm{n}=5$ ). 
blotting, the expression level of Prx IV was low in all tissue samples including cancer tissue. Altogether, these results suggest that Prx IV is not directly involved in cervical cancer development (Figs. 2 and 3).

\section{Discussion}

Many studies have been conducted on peroxiredoxin I (Prx I) in various kinds of human carcinoma tissues. Peroxiredoxin I (Prx I) was overexpressed in several cancer types, including carcinomas of the thyroid gland, breast, and non-small cell lung cancer (NSCLC) tissue (7,11-13). Furthermore, it was reported that expression of peroxiredoxins, especially III, IV and $\mathrm{V}$, is increased in breast malignancy, suggesting the induction of Prxs in response to increased production of reactive oxygen species in carcinomatous tissue (8). These reports suggest that Prx family has a close relationship with cancer development. However, scarce data are available on the Prx family in cervical cancer. Therefore, we evaluated the expression patterns of the Prx family in 202 tissue samples by immunohistochemistry. In order to monitor the expression levels of the Prx family in cervical cancer, immunoblotting with Prx antibodies were performed with the cervical cancer tissue samples. In this study, we focused on the four mammalian 2-Cys members (Prx I-IV), one of two major Prx subfamilies that utilize thioredoxin as the electron donor for antioxidation. We monitored the expression levels of the Prx Family in conjunction with the cervical cancer development stages.

Previous studies have suggested that Prx I can be used as a tumor marker in many type of cancer cells (11). In the present study, Prx I expression was negative or moderately positive in normal tissues. High-grade CINs and $\mathrm{Cx}$ ca also showed weak Prx I expressions, suggesting that Prx I may not be a tumor marker at least in cervical cancer. This discrepancy is probably related to the nature of cervical cancer. In line with this observation, Prx I isoform was expressed not only in the cancer cells, but also in numerous non-cancer cells, such as alveolar macrophages (14).

Our data showed that Prx II and Prx III were clearly elevated in most cervical cancer cells as assessed by immunoblotting. Interestingly, Prx II and Prx III were highly expressed in high-grade CINs and Cx ca, while undetectable or weakly expressed in normal and low-grade CINs, suggesting that they can be used as a tumor marker to predict progression of cervical cancer. There are several lines of evidence in other types of cancer in support of this proposal. It has been reported that Prx II can be used a novel marker of vascular tumors (15) and that the enhanced expression of Prx III might be associated with the formation and development of hepatocellular carcinomas (16).

Karihtala et al showed that all of the Prx isoforms were found in the majority of breast cancer species, with the most intense staining being of Prx IV (8). However, in the present study, immunohistochemistry and immunoblotting experiments did not show any noticeable difference among cervical specimens. As described previously, this discrepancy may be due to the difference between breast cancer and cervical cancer.
For the first time, we showed by immunohistochemistry that Prx II and Prx III are upregulated in reponse to cervical cancer development. We tried to confirm these results by immunoblotting. However, the results of immunoblotting and immunohistochemisty rarely exactly matched each other, since cell-specific expression can be more easily and precisely assessed by immunohistochemistry, while immunoblotting analysis is less sensitive for cell-specific changes from homogenates. We reason that cervical cancer tissue specimens might be contaminated with surrounding normal tissue.

Our results suggest Prx II and Prx III are the key molecules associated with cervical cancer. Understanding the function and biological role of these Prx isoforms may lead to important discoveries on the cellular dysfunction of malignant epithelial cervical cancer, and furthermore may be applied in the development of therapeutic agents for patients who have resistance to cancer therapy.

\section{Acknowledgments}

This research was supported by the Kyung Hee University Research Fund in 2005 (KHU-20051039) and the Korea Science and Engineering Foundation (KOSEF) grant funded by the Korea government, Ministry of Science Technology [No.R13-2002-020-02003-0(2008)].

\section{Reference}

1. Notani PN: Global variation in cancer incidence and mortality. Curr Sci 81: 465-474, 2001

2. Kim K, Kim IH, Lee KY, et al: Stadtman: the isolation and purification of a specific 'protector' protein which inhibits enzyme inactivation by a thiol/Fe(III)/O2 mixed-function oxidation system. J Biol Chem 263: 4704-4711, 1988.

3. Chae HZ, Kim IH, Kim K, et al: Cloning, sequencing, and mutation of thiol-specific antioxidant gene of Saccharomyces cerevisiae. J Biol Chem 268: 16815-16821, 1993.

4. Naidu MK, Suryakar AN, Swami SC, et al: Oxidative stress and antioxidant status in cervical cancer patients. IJCB 22: 140-144, 2007.

5. Butterfield LH, Merino A, Golub SH, et al: From cytoprotection to tumor suppression: the multifactorial role of peroxiredoxins. Antioxid Redox Signal 1: 385-402, 1999.

6. Lehtonen ST, Svensk AM, Soini Y, et al: Peroxiredoxins, a novel protein family in lung cancer. Int J Cancer 111: 514-521, 2004.

7. Chang JW, Lee SH, Jeong JY, et al: Peroxiredoxin-I is an autoimmunogenic tumor antigen in non-small cell lung cancer. FEBS Lett 579: 2873-2877, 2005.

8. Karihtala P, Mäntyniemi A, Kang SW, et al: Peroxiredoxins in breast carcinoma. Clin Cancer Res 9: 3418-3424, 2003.

9. Park JH, Kim YS, Lee HL, et al: Expression of peroxiredoxin and thioredoxin in human lung cancer and paired normal lung. Respirology 11: 269-275, 2006.

10. Kim YJ, Ahn JY, Liang P, et al: Human prx 1 gene is a target of Nrf2 and is up-regulated by hypoxia/reoxygenation: implication to tumor biology. Cancer Res 67: 546-555, 2007.

11. Yanagawa T, Iwasa S, Ishii T, et al: Peroxiredoxin I expression in oral cancer: a potential new tumor marker. Cancer Lett 156: 27-35, 2000.

12. Yanagawa $\mathrm{T}$, Ishikawa $\mathrm{T}$, Ishii $\mathrm{T}$, et al: Peroxiredoxin I expression in human thyroid tumors. Cancer Lett 145: 127-132, 1999.

13. Chang JW, Jeon HB, Lee JH, et al: Augmented expression of peroxiredoxin I in lung cancer. Biochem Biophys Res Commun 289: 507-512, 2001.

14. Conway JP and Kinter M: Dual role of peroxiredoxin I in macrophage-derived foam cells. J Biol Chem 281: 27991-28001, 2006.

15. Lee SC, Na YP, Lee JB, et al: Expression of peroxiredoxin II in vascular tumors of the skin: a novel vascular marker of endothelial cells. J Am Acad Dermatol 49: 487-491, 2003. 
16. Choi JH, Kim TN, Kim S, et al: Overexpression of mitochondrial thioredoxin reductase and peroxiredoxin III in hepatocellular carcinomas. Anticancer Res 22: 3331-3335, 2002.

17. Seo MS, Kang SW, Kim K, et al: Identification of a new type of mammalian peroxiredoxin that forms an intramolecular disulfide as a reaction intermediate. J Biol Chem 275: 20346-20354, 2000.

18. Hofmann B, Hecht HJ and Flohé: Peroxiredoxin. Biol Chem 383: 347-364, 2002.

19. Immenschuh S and Baumgart-Vogt E: Peroxiredoxins, oxidative stress and cell proliferation. Antioxid Redox Signal 7: 768-777, 2005.

20. Banmeyer I, Marchand C, Clippe A, et al: Human mitochondrial peroxiredoxin 5 protects from mitochondrial DNA damages induced by hydrogen peroxide. FEBS Lett 579: 2327-2333, 2005.

21. Noh DY, Ahn SJ, Lee RA, et al: Overexpression of peroxiredoxin in human breast cancer. Anticancer Res 21: 2085-2090, 2001
22. Yanagawa $\mathrm{T}$, Ishikawa $\mathrm{T}$, Ishii $\mathrm{T}$, et al: Peroxiredoxin I expression in human tyroid tumors. Cancer Lett 145: 127-132, 1999.

23. Neumann CA, Krause DS, Carman CV, et al: Essential role for the peroxiredoxin $\operatorname{Prdx} 1$ in erythrocyte antioxidant defence and tumour suppression. Nature 424: 561-565, 2003.

24. Kinnula VL, Lehtonen S, Kaarteenaho-Wiik R, et al: cell specific expression of peroxiredoxins in human lung and pulmonary sarcoidosis. Thorax 57: 157-164, 2002.

25. Kim JH, Bogner PN, Ramnath N, et al: Elevated peroxiredoxin 1, but not NF-E2-related factor 2 , is an independent prognostic factor for disease recurrence and reduced survival in stage I non-small cell lung cancer. Clin Cancer Res 13: 3875-3882, 2007.

26. Kinnula VL, Lehtonen S, Sormunen R, et al: Overexpression of peroxiredoxins I, II, III, V, and VI in malignant mesothelioma. J Pathol 196: 316-323, 2002 